\title{
Aplicación de un Esquema de Arquitectura Empresarial (TOGAF) para una Pequeña Empresa (PYME) utilizando Aplicaciones Colaborativas de Google
}

\author{
Rosmery Canabal, Amaury Cabarcas y Raúl J. Martelo \\ Universidad de Cartagena, Facultad de Ingeniería, Grupo de Investigación en Tecnologías de las \\ Comunicaciones e Informática, GIMATICA, Avenida del Consulado, Calle 30, No. 48 - 152, Cartagena- \\ Colombia. (e-mail: rcanabal@unicartagena.edu.co, acabarcasa@unicartagena.edu.co, \\ rmartelog1@unicartagena.edu.co).
}

Recibido Ago. 31, 2016; Aceptado Oct. 28, 2016; Versión final Dic. 28, 2016, Publicado Ago. 2017

\begin{abstract}
Resumen
Éste artículo presenta el diseño de una arquitectura empresarial soportada en la Web 2.0 para una pequeña empresa (PYME) del sector metalmecánico en la ciudad de Cartagena, Colombia. Para el diseño de la arquitectura empresarial se emplearon las seis primeras fases de la metodología que proporciona el método de arquitectura empresarial TOGAF. El artículo presenta la arquitectura diseñada usando las fases de TOGAF que permitirían a la PYME lograr el cumplimiento de los objetivos estratégicos del negocio, alineándolos a las herramientas colaborativas de Google. Se concluyó que el uso de las buenas prácticas que proporciona la metodología TOGAF, permite determinar que la PYME está en la capacidad de establecer, asignar roles, tareas y procesos específicos soportados en herramientas de la Web 2.0 y el uso de las herramientas colaborativas como las de Google soportadas en la nube, permitiendo que a bajo costo las Pymes puedan contar con estrategias orientadas al uso y aplicación de tecnologías de la información en los procesos del negocio
\end{abstract}

\section{Application of an Open Group Architecture Framework (TOGAF) to a Small Enterprise (SME) using Google Collaborative Applications}

\begin{abstract}
This article presents the design of a business architecture supported in Web 2.0 for a small enterprise (SME) in the metalworking sector in the city of Cartagena, Colombia. For the design of the business architecture, the first six phases of the TOGAF architecture framework methodology. The article presents the architecture designed using the TOGAF phases that would allow the SME to achieve the strategic objectives of the business by aligning them with Google's collaborative tools. It is concluded that the use of the good practices provided by the TOGAF methodology, allows determining the ability of the SME to establish, assign specific roles, tasks and processes supported in Web 2.0 tools. In addition, the use of collaborative tools such as those of Google supported in the cloud, are a low cost alternative for any SME to have strategies for using information technology in its business processes
\end{abstract}

Keywords: enterprise architecture; TOGAF; Web 2.0; SME; metalworking sector 


\section{INTRODUCCIÓN}

En Colombia en los últimos años, el gobierno, la academia y el sector privado, han dirigido sus estrategias de apoyo y sus servicios hacia todos los sectores industriales en especial a las pequeñas y medianas empresas (Pymes), pero particularmente a las del sector metalmecánico. A nivel nacional e internacional se han planteado alternativas a los problemas de las pymes a la hora de implementar herramientas tecnológicas tanto de hardware como software en diversas áreas. Ejemplos de estas soluciones se pueden encontrar en países como Colombia en agencias de viajes implementando tecnologías de comunicaciones de voz sobre la IP (Martelo et al., 2015), en el desarrollo de sistemas de información en pymes para apoyar la toma de decisiones (Cabarcas et al., 2015). En otros países como Venezuela existen estudios orientados a identificar las limitaciones en pymes agroalimentarias que no les permiten mejorar su participación competitiva (Cardozo et al., 2011). Se han podido identificar obstáculos, que estas pequeñas y medianas empresas tienen para su desarrollo y para mejorar su participación competitiva, en el caso de Colombia se podrían mencionar las dificultades en la identificación y acceso a la tecnología adecuada, la formalización y absorción de nuevas tecnologías, entre otros (Hernández, et al., 2014).

Es claro que para poder solucionar estos obstáculos y mejorar la competitividad es de suma importancia que las pymes implementen de manera adecuada herramientas tecnológicas acordes a los objetivos del negocio. Los beneficios potenciales al implementar herramientas tecnológicas en una organización son muchos, dentro de los cuales se incluyen desde los de carácter operativo, como el aumento de la eficiencia automatizando procesos, hasta los beneficios de carácter estratégico, como la mejora de los procesos empresariales. Si bien es cierto que las pymes pueden mejorar la eficiencia de los diferentes procesos empresariales con la ayuda de las Tecnologías de la Información (TI), se debe tener en cuenta que el éxito radica en el uso que se le da a dichas tecnologías. Es claro que no basta sólo con implementarlas, por lo tanto hoy en día es de vital importancia la adopción y el enfoque de la arquitectura empresarial $(\mathrm{AE})$, con el fin de encontrar procesos innovadores que permitan diseñar y asegurar un desempeño alineado a los objetivos estratégicos de una organización o empresa con las TI. El uso de AE lleva a definir un verdadero plan estratégico de la organización, teniendo en cuenta los componentes de negocio y los sistemas de información, además permite conocer el estado ideal al que podría llegar la organización, a través de las tecnologías que soportan los procesos necesarios para alcanzarlo. A nivel internacional existen varios casos que permiten evidenciar los beneficios y las mejoras en las organizaciones al diseñar e implementar AE, es así como se pueden destacar los esfuerzos realizados en países como Malasia donde agencias del sector público realizan esfuerzos para mejorar las alternativas en e-Gobierno (Bakar, et al., 2016) igualmente hay otros referentes como (Närman, et al, 2016), enfocados a medir los impactos del uso de AE en las organizaciones. A nivel latinoamericano podemos encontrar el uso de $A E$ en el Banco de Estado en Ecuador (Salazar y Heyl, 2016) enfocado a rediseñar sus procesos de negocios.

En Colombia las apuestas en el uso de AE parten de los entes de gobierno, como es el caso del Ministerio de Tecnologías de la Información y las Comunicaciones, donde desde el año 2014 se presentó un borrador de $A E$ para el gobierno Colombiano, el proyecto pretende un Estado mejor articulado que transforme la gestión pública, a través de las tecnologías de la información y las comunicaciones (TIC) y simplifique la vida de los ciudadanos (MINTIC, 2014). Es así que actualmente Colombia cuenta con un Marco de Referencia de AE como principal instrumento para implementar la Arquitectura TI y habilitar la Estrategia de Gobierno en línea. Con él se busca habilitar las estrategias de las TIC para servicios, para la gestión, para el gobierno abierto y para la seguridad y la privacidad. (MINTIC, 2016)

Actualmente, las organizaciones se enfrentan a un entorno dinámico y regido bajo un contexto de globalización, así como a la necesidad de mantener altos niveles de competitividad. El rápido avance tecnológico ha propiciado en algunas organizaciones mayor eficiencia empresarial, originando una mayor facilidad y velocidad para el acceso, procesamiento y difusión de la información. Sin embargo, según una investigación del centro internacional de investigaciones para el desarrollo, en colaboración con el fondo multilateral de inversiones y el banco interamericano de desarrollo llamada "Las TIC en el desarrollo de la Pyme", las empresas que no utilizan las TI como herramientas competitivas en sus negocios, limitan su crecimiento empresarial y podrían perder hasta $30 \%$ de sus ingresos (Ca' Zorzi, 2011). A pesar de lo anterior, la absorción de las TI por parte de las empresas más pequeñas no ha sido uniforme, tampoco los beneficios de este tipo de inversiones han sido demostrados en forma convincente. A pesar de esto, el impacto de estas tecnologías es visible en todas las áreas de las empresas, la importancia de las TI crece con el tiempo y sus efectos están visibles para todos (Slusarczyk et al., 2015). Es así que desde esta perspectiva, las Pymes colombianas deben alinearse con las tendencias que impone la gerencia del siglo $\mathrm{XXI}$, en donde la competitividad se convierte en el eje central del negocio y la producción de conocimiento, en el factor determinante para transformar este tipo de empresas y estimular la creatividad, la generación de conocimientos y promover la creación de productos y servicios con valor agregado y pertinencia social (Villegas 2010). Esto es válido para todos los sectores industriales, pero particularmente para el 
metalmecánico, por su papel impulsor para el desarrollo de otros sectores de la economía. Ante la necesidad presentada anteriormente, se consideró relevante adelantar estrategias para el diseño de una AE en una pyme que le permita alinear las tecnologías de la información con los objetivos de negocio y le permita incluir la Web 2.0 como herramienta tecnológica. Para el diseño de la AE donde se puedan incluir estas herramientas tecnológicas se debe tener en cuenta el tipo de empresa y cada una de las necesidades individuales, en este artículo se aborda el caso de la empresa cartagenera "La casa del embobinador", la cual ofrece servicios de mantenimiento y reparación de máquinas eléctricas.

Éste artículo presenta el diseño de una arquitectura empresarial usando el marco de trabajo de arquitectura de Open Group (The Open Group Architecture Framework, TOGAF por sus siglas en inglés) (The Open Group, 2011) y el método de desarrollo de la arquitectura (ADM por sus siglas en inglés) en sus seis primeras fases (preliminar, visión de arquitectura, arquitectura del negocio, arquitectura sistemas de información, arquitectura de tecnológica y oportunidades y soluciones), dando como resultado el diseño de arquitectura empresarial soportada en la Web 2.0.

\section{METODOLOGÍA}

La metodología usada hace referencia al núcleo de TOGAF, la cual es el método de desarrollo de la arquitectura (ADM por sus siglas en inglés) y las técnicas usadas en cada fase (The Open Group, 2011). La metodología utilizada está enfocada en el método núcleo (ADM) de TOGAF y los diferentes artefactos de cada una de sus fases. En este caso particular, la selección de TOGAF responde a las preferencias personales de los autores y al conocimiento de los mismos sobre el framework y su metodología, sumado a su creciente popularidad en el ámbito empresarial (Bloomberg 2014). Adicional a eso se destacan los potenciales beneficios que su aplicación tiene dentro de los que encontramos, la gestión eficiente y la exploración de las capacidades que existen dentro de la empresa y segundo, busca la alineación de las tecnologías de la información (TI) con la visión estratégica, las metas y objetivos. Y por último se destacan dos tipos de beneficios, el método provee consistencia entre los procesos y la información con las unidades de negocio y permite mejorar la confiabilidad y reducción de riesgos. Y los beneficios de tecnologías de la información (TI) que permitirá incrementar la eficiencia del negocio con las operaciones de TI. (The Open Group, 2011).

Cabe resaltar que la investigación tiene alcance hasta la fase de oportunidades y soluciones, dado que el diseño no ha sido implementado ni validado con las fases restantes. Con la propuesta o aplicación de este método dentro de la empresa objeto de caso de estudio, se busca plantear estrategias a la optimización del proceso principal del negocio el cual es la gestión de la orden interna de trabajo (OIT) y la alineación del mismo con las tecnologías de la información para incrementar la eficiencia y la competitividad de la empresa. El ADM es un método para el desarrollo y gestión del ciclo de vida de una arquitectura empresarial que puede ser usada por cualquier empresa, ayuda a conocer el negocio y las necesidades de TI de la organización, la metodología involucra a los interesados en diferentes niveles y en diferentes fases. Tiene una amplia aplicación a través de las organizaciones, ya que permite la utilización de otros frameworks que pueden brindar solución a sus necesidades (The Open Group, 2011). Para el caso específico abordado en este artículo solo se tomaron en cuenta seis fases: preliminar, visión de arquitectura, arquitectura del negocio, arquitectura de sistemas de información, arquitectura tecnológica y la fase de oportunidades y solución, es importante aclarar que en cada fase se utilizaron las guías y/o técnicas necesarias para la realización de los catálogos y/o diagramas por ejemplo el análisis de brechas (Postina et Al 2009).

\section{RESULTADOS}

Como resultado de la investigación se relacionan las fases trabajadas y mencionadas en la metodología: fase preliminar, visión de arquitectura, arquitectura del negocio, arquitectura de sistemas de información, arquitectura tecnológica y la fase de oportunidades y solución.

\section{Fase preliminar}

Esta fase tiene como objetivo determinar y establecer la capacidad deseada de la organización a través de la técnica de principios del negocio y de arquitectura. En esta fase se tuvo en cuenta el modelo organizacional de la empresa, a partir de entrevistas y visitas al personal administrativo. A través de la identificación de la organización de la pyme y los principios del negocio se logró determinar los planes y estrategias del modelo motivacional, dentro de los cuales se pudo evidenciar la poca utilización de herramientas TI como apoyo en el proceso principal. Se estableció que la capacidad del negocio está enfocada en la reparación de máquinas de corriente directa y alterna y de motores eléctricos. Igualmente se identificó que la brecha tecnológica asociada al proceso de gestión de la OIT es alta, dado que el proceso 
mencionado se da en gran parte de forma manual y el porcentaje de interacción con las partes interesadas después de la solicitud es bajo. En cuanto a las partes interesadas (gerencia, producción, clientes, socios, logística) participaron en la especificación de requerimientos y principios de la pyme como: la importancia de la relación con clientes y proveedores y la confiabilidad en el uso de $\mathrm{TI}$ en el proceso principal y permitieron identificar que el acceso a la información debe ser protegido. Como resultado nace la solicitud de trabajo de arquitectura, la cual contiene, las metas y cambios del negocio, planes estratégicos del negocio, restricciones organizacionales, descripción del sistema actual del negocio y la descripción actual de sistema de arquitectura y de TI.

\section{Fase A: Visión de arquitectura}

En esta fase se identificaron los roles y responsabilidades de las partes interesadas, así como las preocupaciones tales como: la optimización del proceso, la disminución de costos, la no utilización de buenas prácticas y la mejora en la utilización y efectividad de TI por medio de la web 2.0 en el proceso de gestión de la OIT. Lo anterior dio lugar a la aplicación de la técnica de escenario de negocio (The Open Group, 2011) que permitió definir el alcance y las expectativas de la pyme con respecto a el desarrollo de la arquitectura en donde se encontraron los siguientes escenarios: 1. Para incrementar el grado de eficiencia y competitividad en los procesos de la pyme, se propuso mejorar en un $20 \%$ el número de orden interna de trabajo (OIT) atendidas mensualmente y 2. Para la búsqueda de herramientas de la web 2.0 que apoyen los objetivos del negocio, se tuvo como alcance incrementar en un 30\% la utilización de las herramientas de google drive para apoyar los procesos internos de la OIT en un periodo no mayor a un año. Estos escenarios permitirán definir más adelante los nuevos indicadores de gestión.

\section{Fase B: Arquitectura del Negocio}

Para la obtención de los resultados de esta fase se tuvo en cuenta los principios del negocio y las capacidades evaluadas en la visión de arquitectura (Fase A). El objetivo de esta fase consistió en desarrollar la arquitectura objetivo (target) de la fase b, que describa como la empresa necesita operar para alcanzar las metas del negocio. Para esto, toma importancia el plan de comunicación de la empresa, lo que llevo a que se hiciera un análisis del plan de comunicación de la gestión de la OIT, y se encontró un nivel de eficiencia y eficacia bajo, dado que los mecanismos de retroalimentación no permiten cumplir con lo propuesto en la fase A. Para identificar los componentes candidatos de la hoja de ruta de las actividades desde la arquitectura actual hasta la objetivo, se utilizó la técnica "Análisis de Brechas" o análisis de deficiencias, ya que es ampliamente usada por el ADM para validar la arquitectura que se está desarrollando (Postina et Al 2009). En la tabla 1 se muestra las diferentes brechas que se deben tener en cuenta para llegar al objetivo, también se realizó el diagrama que se muestra en la figura 1, sobre la Orden Interna de Trabajo (OIT) donde se puede observar el flujo de la gestión de la OIT.

Tabla 1: Análisis de brechas de la fase b: Arquitectura del negocio

\begin{tabular}{|c|c|c|c|c|c|c|c|}
\hline \multirow[b]{2}{*}{$\begin{array}{l}\text { Línea base de } \\
\text { la arquitectura }\end{array}$} & \multicolumn{7}{|c|}{ Arquitectura Objetivo } \\
\hline & $\begin{array}{l}\text { Servicio } \\
\text { de } \\
\text { solicitud }\end{array}$ & $\begin{array}{c}\text { Servicio } \\
\text { de } \\
\text { revisión }\end{array}$ & $\begin{array}{l}\text { Servicio de } \\
\text { cotización }\end{array}$ & $\begin{array}{l}\text { Servicio de } \\
\text { gestionar } \\
\text { requisición }\end{array}$ & $\begin{array}{l}\text { Servicio de } \\
\text { gestión de } \\
\text { producción }\end{array}$ & $\begin{array}{c}\text { Servicio } \\
\text { de } \\
\text { entrega }\end{array}$ & $\begin{array}{l}\text { Servicios } \\
\text { eliminados }\end{array}$ \\
\hline $\begin{array}{l}\text { Solicitud del } \\
\text { servicio }\end{array}$ & Incluido & & & & & & \\
\hline $\begin{array}{l}\text { Revisión del } \\
\text { servicio }\end{array}$ & & incluido & & & & & \\
\hline $\begin{array}{l}\text { Servicio de } \\
\text { cotización }\end{array}$ & & & Omitido & & & & \\
\hline $\begin{array}{l}\text { Servicio de } \\
\text { requisición }\end{array}$ & & & & omitido & & & \\
\hline Producción & & & & & Omitido & & \\
\hline $\begin{array}{l}\text { Servicio de } \\
\text { entrega }\end{array}$ & & & & & & incluida & \\
\hline Nuevo & & & $\begin{array}{l}\text { Brecha: enlazar } \\
\text { la cotización con } \\
\text { proceso } \\
\text { colaborativo de } \\
\text { herramientas de } \\
\text { Google drive }\end{array}$ & $\begin{array}{l}\text { Brecha: enlazar la } \\
\text { requisición con } \\
\text { proceso } \\
\text { colaborativo de } \\
\text { herramientas de } \\
\text { Google drive }\end{array}$ & $\begin{array}{l}\text { Brecha: enlazar } \\
\text { la gestión con } \\
\text { proceso } \\
\text { colaborativo de } \\
\text { herramientas de } \\
\text { Google drive }\end{array}$ & & \\
\hline
\end{tabular}




\section{Fase C: Arquitectura de sistemas de información (Datos - Aplicación)}

En esta fase, al igual que en la fase B se aplicó la técnica definida anteriormente con el objetivo de identificar las deficiencias y desarrollar el sistema de información deseado (datos - aplicación). En la tabla 2 se describe como la fase $\mathrm{C}$ permitirá que la arquitectura del negocio y la visión de arquitectura estén direccionadas a las preocupaciones de los interesados en cuanto a la solicitud, generación y gestión de la orden interna de trabajo (OIT) de la pyme.

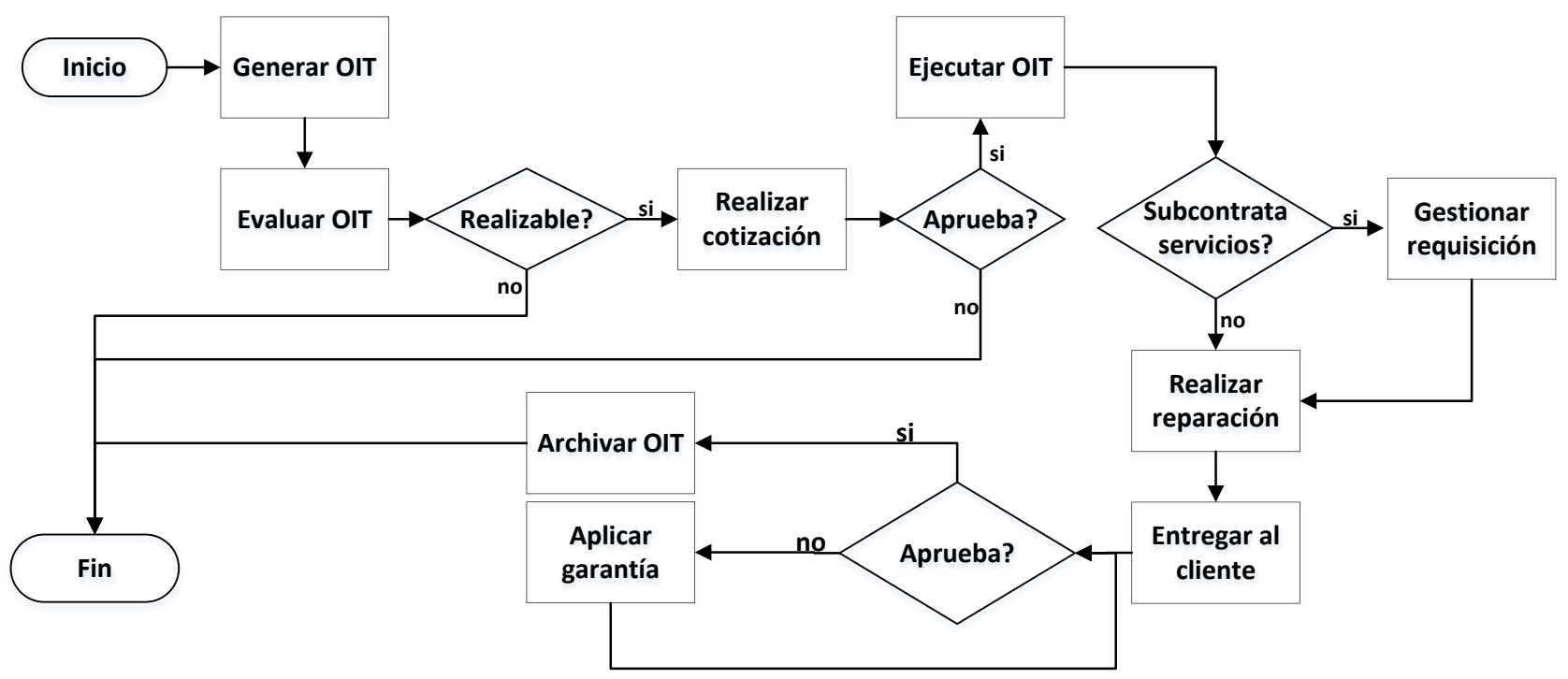

Fig. 1: Diagrama de flujo de servicios e información de negocio de la OIT

Tabla 2: Análisis de brechas de la fase C: arquitectura de sistemas de información

\begin{tabular}{|c|c|c|c|c|c|c|}
\hline \multirow{2}{*}{$\begin{array}{l}\text { Línea base de la } \\
\text { arquitectura: } \\
\text { Entidades de } \\
\text { datos }\end{array}$} & \multicolumn{6}{|c|}{ Arquitectura Objetivo } \\
\hline & $\begin{array}{l}\text { Servicio } \\
\text { de } \\
\text { revisión }\end{array}$ & Servicio de cotización & $\begin{array}{l}\text { Servicio de gestionar } \\
\text { requisición }\end{array}$ & $\begin{array}{l}\text { Servicio de gestión } \\
\text { de producción }\end{array}$ & $\begin{array}{l}\text { Servicio } \\
\text { de } \\
\text { entrega }\end{array}$ & $\begin{array}{l}\text { Servicios } \\
\text { eliminados }\end{array}$ \\
\hline $\begin{array}{l}\text { Orden interna de } \\
\text { trabajo }\end{array}$ & incluido & & & incluido & incluido & \\
\hline $\begin{array}{l}\text { Jefe de } \\
\text { Producción }\end{array}$ & incluido & No definido & Incluido & incluido & incluido & \\
\hline Cliente & & No definido & & No definido & incluido & \\
\hline Mantenimiento & & No definido & & No definido & & \\
\hline Cotización & & No definido & & & & \\
\hline Jefe Comercial & incluido & & & & incluido & \\
\hline Nuevo & & $\begin{array}{l}\text { Brecha: las entidades } \\
\text { pueden consultar la } \\
\text { cotización y aprobar por } \\
\text { medio de herramientas } \\
\text { de Google drive }\end{array}$ & $\begin{array}{l}\text { Brecha: las entidades } \\
\text { pueden gestionar } \\
\text { requisición y aprobar } \\
\text { por medio de } \\
\text { herramientas de } \\
\text { Google drive }\end{array}$ & $\begin{array}{l}\text { Brecha: las } \\
\text { entidades pueden } \\
\text { hacer seguimiento y } \\
\text { aprobar por medio } \\
\text { de herramientas de } \\
\text { Google drive }\end{array}$ & & \\
\hline
\end{tabular}

\section{Fase D: Arquitectura tecnológica}

Para el cumplimiento de esta fase se seleccionó las herramientas colaborativas de Google, teniendo en cuenta que tienen beneficios como la disminución de costos, aumento de la productividad de los empleados a través de la utilización de comunicación y colaboración sencilla y eficiente. Estas herramientas implementan cambios e innovaciones con mayor flexibilidad, ya que la información se podría acceder desde cualquier lugar, en cualquier plataforma y permite el trabajo colaborativo. En este caso las herramientas de Google, permiten disponer de una gran variedad de aplicaciones online tales como Google Calendar, Google Docs, Google Drive, Correo corporativo, entre otras. Todas están vinculadas entre sí para obtener un mejor rendimiento corporativo que permita una mejor implementación del Framework seleccionado (Marston et Al 2011). El objetivo de esta fase fue desarrollar la arquitectura tecnológica objetivo, por medio del uso de la web 2.0 seleccionando las herramientas de Google y sus principales aplicaciones como la tecnología más adecuada para la empresa, como se muestra en la figura 2. 


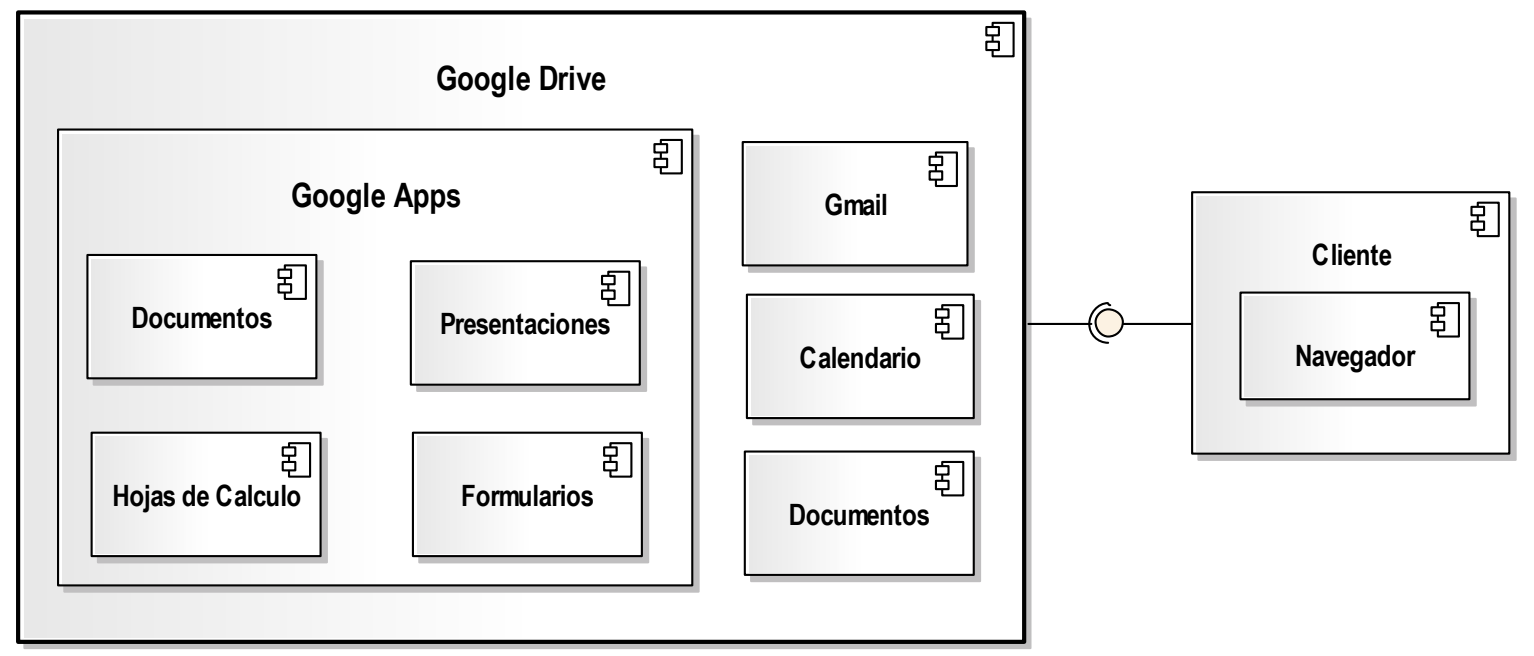

Fig. 2: Diagrama de componentes

El diagrama de procesos de negocio que se observa en la figura 3, representa los procesos o eventos que soporta la arquitectura tecnológica propuesta. La cual consistió en implementar las principales herramientas de Google para la gestión de las OIT, cómo se muestra en la figura 4, de tal forma que los archivos se puedan compartir entre los distintos departamentos de forma colaborativa.

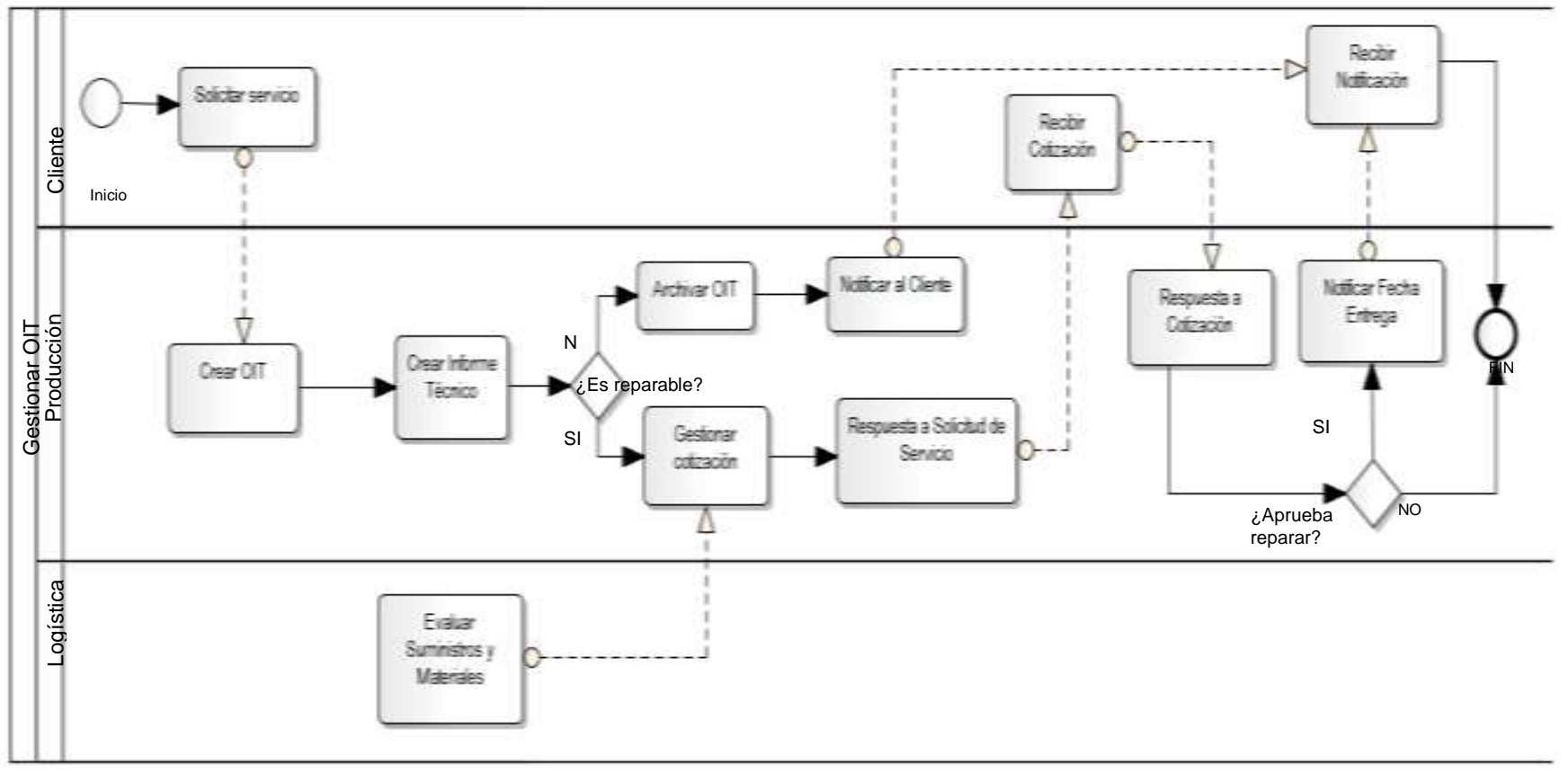

Fig. 3: Diagrama de proceso de negocio para gestionar la OIT

\section{Fase E: Oportunidades y Soluciones}

En esta fase se identificaron las brechas de las fases $B, C$ y $D$, que permitirá migrar hacia una nueva arquitectura empresarial que aporte al mejoramiento de los servicios de la pyme y a la comunicación eficiente de los interesados internos y externos. En la arquitectura propuesta se solicitará el servicio mediante el correo corporativo que está funcionando en la plataforma de Gmail, con la creación de la OIT en la aplicación de formularios de Google, se genera un informe técnico del equipo a reparar y se comparte en simultáneo con todo el personal de producción. La cotización del servicio realizada a través de hojas de cálculo de Google Apps será compartida con el personal de logística para aprobar los costos y suministros que se deben cotizar (requisición). La requisición cuenta con un repositorio en Google Docs donde se deben recibir todas las cotizaciones enviadas por los proveedores. De esta forma, se organiza la información, permitiendo crear una estructura de repositorio con almacenamiento en la nube y así disponer de los archivos de cotización y/o proveedores en línea. Seguido a lo anterior, se comparte la cotización con el cliente a la espera de aprobación, si es aprobada, en Google Calendar se genera la fecha de entrega y se comparte el evento con el personal de cada área administrativa. 


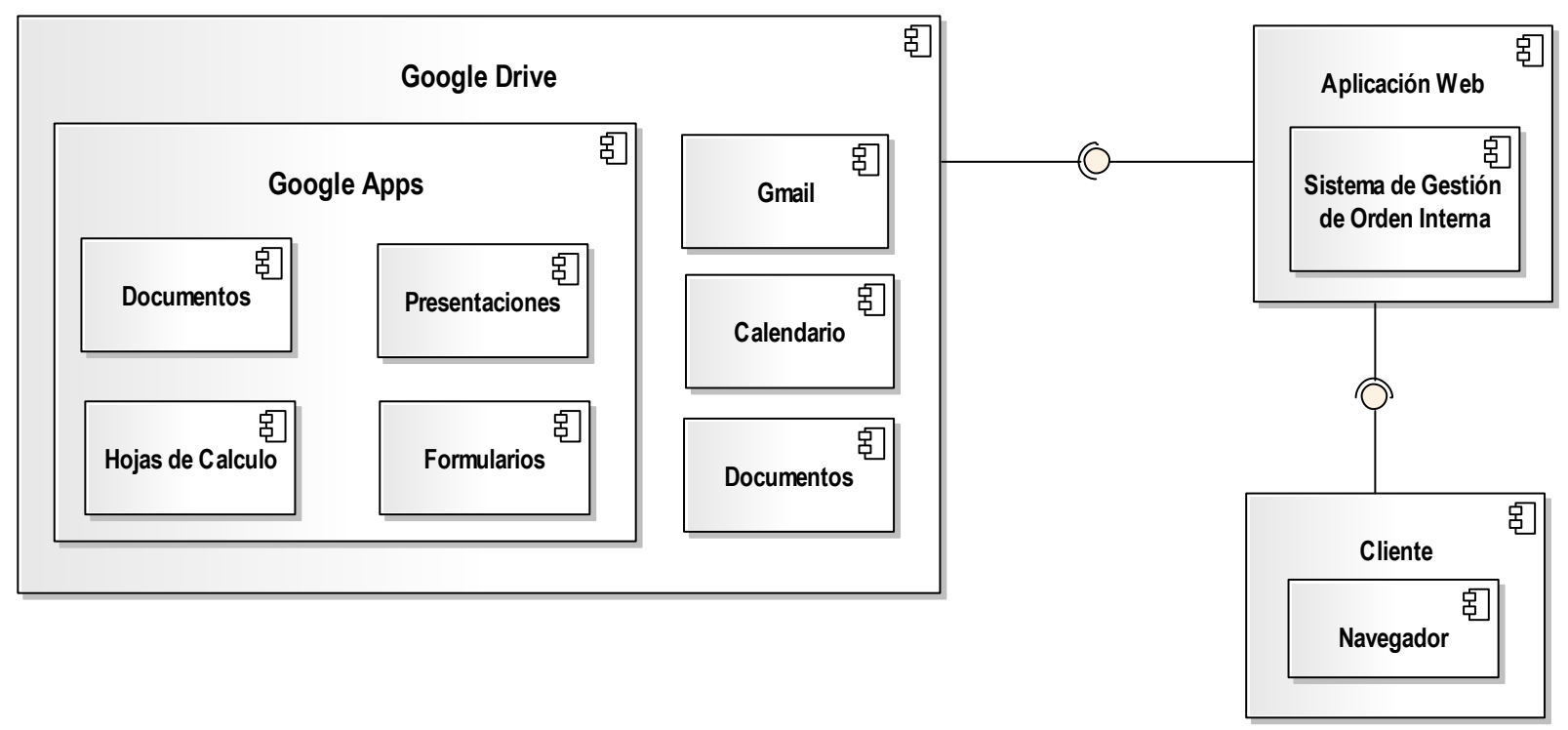

Fig. 4: Diagrama de Sistema de gestión de OIT

\section{CONCLUSIONES}

Las conclusiones del presente trabajo están enfocadas a identificar las lecciones aprendidas de la aplicación del marco de trabajo y el método ADM, a partir de los resultados y de la aplicación del método se pudo concluir lo siguiente: 1) El uso de las buenas prácticas que proporciona la metodología de TOGAF, permite determinar que la pyme está en la capacidad de: establecer, asignar roles, tareas y procesos específicos soportados en herramientas de la web 2.0,2) Con la utilización de TOGAF se ve la empresa como un todo y no como partes independientes, 3) con la metodología que ofrece TOGAF la retroalimentación es constante entre cada fase de ADM, esto permite que se pueda adaptar rápidamente a los cambios, 4) el análisis de brechas es una técnica que permite definir claramente la hoja de ruta entre el estado actual y la arquitectura objetivo, 5) dado que el marco de trabajo TOGAF es de libre acceso, permite la implementación y la posibilidad de combinarse con otros marcos y entes que lo gobiernan con el objeto de poder adaptarse a las necesidades del negocio y los desafíos del entorno, 6) las guías y técnicas que provee el método ADM son un aporte importante que pueden adoptarse y adaptarse con el objeto de mejorar la estrategia empresarial (visión y misión) y en la identificación de las tecnologías dentro de la misma para el logro de los objetivos estratégicos, 7) el uso de las herramientas colaborativas como las de Google soportadas en la nube, son una alternativa para cualquier pyme, que permiten a bajo costo contar con estrategias orientadas al uso y aplicación de TI en los procesos del negocio, 8) se recomienda la validación y posterior implementación de la arquitectura diseñada, junto con el uso de buenas prácticas con el objeto de obtener mejoras significativas en los servicios y procesos que pueden ser soportados en la web 2.0 para disipar la brecha competitiva.

\section{REFERENCIAS}

Bakar, N., Kama, N., y Harihodin, S. Enterprise architecture development and implementation in public sector: The Malaysian perspective. Journal of Theoretical and Applied Information Technology, 88 (1), 176$188(2016)$

Bloomberg, J. Enterprise Architecture: Don't Be a Fool with a Tool. Rev. Forbes, ISSN: 0015 - 6914, 2014, (en línea), https://goo.gl/T10sg6. Acceso: 15 de Mayo (2016)

Cabarcas, A., Puello, P., y Martelo, R.J. Sistema de Información Soportado en Recuperación XML para Pequeñas y Medianas Empresas (Pyme) de Cartagena de Indias, Colombia, doi: 10.4067/S071807642015000200016, Inf. Tecnol., (en línea), 26(2), 135-144 (2015)

Cardozo, E.R., Rodríguez, C., y Guaita, W. Las Pequeñas y Medianas Empresas Agroalimentarias en Venezuela y el Desarrollo Sustentable: Enfoque basado en los Principios de Manufactura Esbelta, doi: 10.4067/S0718-07642011000500006, Inf. Tecnol., (en línea), 22(5), 39-48 (2011)

Ca'Zorzi, A. Las TIC en el desarrollo de la Pyme. Algunas experiencias de América Latina, Centro Internacional de Investigaciones para el desarrollo en colaboración con Fondo Multilateral de Inversiones, (en línea), https://goo.gl/pc18IR. Acceso: 15 de junio (2016) 
Hernández, A., Marulanda, C.E., y López, M. Análisis de Capacidades de Gestión del Conocimiento para la Competitividad de PYMES en Colombia, doi: 10.4067/S0718-07642014000200013, Inf. Tecnol, (en línea), 25 (2), 111 - 122 (2014)

Marston, S., Li, Z., Bandyopadhyay, S., Zhang, J., y Ghalsasi, A. Cloud computing - The business perspective. Decision support systems, ISSN 0167-9236, (en línea), 51(1), 176-189 (2011), https://goo.gl/rbCk7v. Acceso: 30 de Junio (2016)

Martelo, R. J., Blanquicet, I., y Rodríguez, L. Metodología para Seleccionar Aplicaciones de la Tecnología de Comunicaciones Voz sobre la IP (VoIP) para Pequeñas y Medianas Empresas (Pymes): El caso de una Agencia de Viajes, doi: 10.4067/S0718-07642015000600014, Inf. Tecnol., (en línea), 26(6), 121 - 128, (2015)

MINTIC, Ministerio de Tecnologías de la Información y las Comunicaciones, (en línea), https://goo.gl/EOV0JW. Acceso: 23 de junio (2016)

MINTIC, Arquitectura TI COLOMBIA - Ministerio de Tecnologías de la Información y las Comunicaciones, (en línea), https://goo.gl/gMpjT1. Acceso: 15 de mayo (2016)

Närman, P., Johnson, P., y Gingnell, L., Using enterprise architecture to analyse how organisational structure impact motivation and learning, doi: 10.1080/17517575.2014.986211, Journal Enterprise Information Systems, 10(5), 523 - 562 (2016)

Postina, M., Sechyn, I., y Steffens, U., "Gap analysis of application landscapes," doi: 10.1109/EDOCW.2009.5331980, $13^{\text {th }}$ IEEE Enterprise Distributed Object Computing Conference Workshops, $274-281$ (2009)

Salazar, N., y Heyl, B., Integration and Implementation of an EA strategy based operating model with BPM technology-Case Study: Housing credit process, Banco Estado Ecuador, doi: 10.1109/SCCC.2015.7416577, Proceedings - International Conference of the Chilean Computer Science Society SCCC, (en línea), 1 - 8 (2016)

Slusarczyk, A., Pozo, J., y Perurena, L., Estudio de aplicación de las TIC en las PYMES, Rev. 3C Empresa Investigación y pensamiento crítico, ISSN: 2254-3376, (en línea), 4(1), 69 - 87 2015, https://goo.gl/XTYplO. Acceso: 24 de abril (2016)

The Open Group, An Introduction to TOGAF 9.1, $1^{\text {st }}$ Ed, 43 - 56. Van Haren Publishing, Londres Inglaterra (2011)

Villegas, D., y Toro, I. Las pymes: Una mirada a partir de la experiencia académica del MBA REVISTA MBA EAFIT, ISSN: 2215-9487, (en línea), 1, 86-101, 2010, https://goo.gl/aDAWwn. Acceso 20 de mayo (2016) 